\title{
Pedigree transmission disequilibrium test for quantitative traits in farm animals
}

\author{
DING XiangDong ${ }^{\dagger}$, WANG ChongLong ${ }^{\dagger} \&$ ZHANG Qin* \\ Key Laboratory of Animal Genetics, Breeding and Reproduction of Ministry of Agriculture, National Engineering Laboratory for Animal \\ Breeding, College of Animal Science and Technology, China Agricultural University, Beijing 100193, China
}

Received February 3, 2012; accepted April 17, 2012

\begin{abstract}
General pedigrees are very common in farm animals, and the recent availability of large panels of SNPs in domestic species has given new momentum to the search for the mutations underlying variation in quantitative traits. In this paper, we proposed a new transmission disequilibrium test approach, called the pedigree transmission disequilibrium test, which deals with general pedigrees and quantitative traits in farm animals. Compared with the existing pedigree disequilibrium test (PDT) and general linear model-based method QTDT, our approach performed better with higher power and lower type I error, especially in scenarios where the quantitative trait locus (QTL) effect was small. We also investigated the application of our approach in selective genotyping design. Our simulation studies indicated that it was plausible to implement a selective genotyping strategy in the proposed pedigree transmission disequilibrium test. We found that our approach performed equally well or better when only some proportion of the individuals in the two tails were genotyped compared with its performance when all the individuals in the pedigree were genotyped.
\end{abstract}

quantitative trait, pedigree transmission, disequilibrium test, association study, gene mapping

Citation: Ding X D, Wang C L, Zhang Q. Pedigree transmission disequilibrium test for quantitative traits in farm animals. Chin Sci Bull, 2012, 57: 2688-2694, doi: $10.1007 / \mathrm{s} 11434-012-5218-8$

The recent availability of high density SNP panels in domestic species has given new momentum to the search for mutations underlying variations in quantitative traits through genome-wide association (GWA) studies. Population-based and family-based designs are commonly used in genetic association studies. Family-based designs have unique advantages over population-based designs because they are robust against population admixture and stratification, allow both linkage and association to be tested, and offer a solution to the problem of model building [1].

A classical family-based association test, the transmission disequilibrium test (TDT), was first proposed by Spielman et al. [2], for disease traits in studies of nuclear families with one affected offspring and two parents. The idea of the TDT is to compare the number of times an allele

$\dagger$ These authors contributed equally this work.

*Corresponding author (email: qzhang@ cau.edu.cn) of interest is transmitted from heterozygous parents to the affected offspring with the number of times other alleles are transmitted from these same heterozygous parents to the affected offspring. The TDT has been extended for use in studies of families with multiple affected offspring [3] and families with only one parent available [4,5]. Most of these methods have the common feature of comparing alleles transmitted with alleles not transmitted from parents to the affected offspring or with alleles transmitted to the unaffected offspring. A limitation of these tests is that, although they remain valid tests of linkage, they are not valid tests of association if related nuclear families and/or sibs from larger pedigrees are used [6]. Larger pedigrees include more information on population substructure than nuclear families, and a test using larger pedigrees can use all potentially informative data, including related nuclear families and discordant sibs. On this basis, Martin et al. [6], proposed the pedigree disequilibrium test (PDT) approach to deal with 
general pedigrees, but, until now, it has only been applied to dichotomous traits.

Most traits that are important in agriculture, medicine and evolution are complex or quantitative traits. Several approaches have been developed to extend the TDT to handle quantitative traits [7-10]. Most of these approaches convert a continuous trait into a dichotomous one. For example, Allison [7] classified all offspring into two groups according to whether or not they received the allele of interest from the heterozygous parent and tested the association by comparing the averages of these two groups. Rabinowitz [8] used a constant to divide the offspring into two groups and an index variable to define the allele transmitting from parents, and constructed a test statistic for data of trios. However, these methods cannot deal with the general pedigrees that are very common in farm animals.

In this paper, we present a pedigree disequilibrium test for quantitative traits, termed the QPTDT, which can deal with general pedigrees, especially those in farm animals. First, the quantitative traits were classified into two categories using two selection criteria for full-sib families and mixed families, and then, a kernel approach similar to, but theoretically superior to the PDT [6], was used. Unlike the PDT, which only uses the information of affected offspring, the QPTDT approach makes use of both the affected and unaffected offspring. When the affected and unaffected offspring are both used, the unaffected offspring also provide transmission information that can increase the power of the test $[11,12]$.

In the following, we first describe the QPTDT approach and then report the results of a simulation study demonstrating its performance compared with the existing QTDT [10] and PDT [6] methods, particularly with respect to the power of the test and type I error. The simulations also demonstrate how well our approach works in selective genotyping design.

\section{Methods}

\subsection{Data simulation}

(i) Pedigree. The base population consisted of 20 males and 80 females who were unrelated to each other. Each male mated randomly with four females and each female produced four offspring with equal probability of being male or female. A second scenario in which each female produced eight offspring was also simulated. Afterward the initial simulation, ten non-overlapped generations were simulated. In each of the generations, 20 males and 80 females were selected at random as parents of the next generation. The mating strategy and the number of offspring per female were the same as in the base population, but mating between half- and full-sibs was avoided. Only the last three generations were used for analysis and the individuals in generation 8 were assumed to be unrelated and without parental information. The populations in the last three generations consisted of a series of independent three-generation pedigrees, each cored with a grandsire as illustrated in Figure 1. In generation 9, a female may or may not have offspring with a probability of 0.5 . In each pedigree, the marker genotypes of one of the parents of a family in the previous generation, which is from another pedigree, may be missing with a probability of 0.3 .

(ii) Genotype and phenotype. A single quantitative trait was simulated following the genetic mixed model, $y=g_{\mathrm{QTL}^{+}}+$ $g_{\text {ploy }}+e$, where $y$ is the phenotypic value, $g_{\mathrm{QTL}}$ is the quantitative trait locus (QTL) genotypic value, $g_{\text {ploy }}$ is the residual polygenic effect following $N\left(0, \sigma_{g}^{2}\right)\left(\sigma_{g}^{2}\right.$ is the additive polygenic variance), and $e$ is a random environmental effect following $N\left(0, \sigma_{e}^{2}\right)$ ( $\sigma_{e}^{2}$ is the residual variance). The QTL genotypic values were derived from Falconer's model [13], $\sigma_{q}^{2}=2 p q a^{2} \quad$ (where $\sigma_{q}^{2}$ is the QTL additive variance, $p$ and $q$ are the allele frequencies at the QTL, and $a$ is the average effect of gene substitution). The minor allele frequency $p$ was set at 0.1 and 0.5 in the base population. Assuming no dominance at the QTL, the genotypic values of the three QTL genotypes QQ, Qq and qq are $a, 0$, and $-a$, respectively.

The genotypes of the offspring were generated according to the genotypes of their parents. The polygenic effect of offspring $i$ with sire $s$ and dam $d$ was generated by $g_{i}=0.5 g_{i}^{s}+0.5 g_{i}^{d}+m_{i}$, where $g_{i}^{s}$ and $g_{i}^{d}$ represent the polygenic value of the offspring's sire and dam, respectively, and $m_{i}$ represents the Mendelian sampling deviation which follows a normal distribution $\mathrm{N}\left(0, \sigma_{m}^{2}\right)\left(\sigma_{m}^{2}=\right.$ $0.25\left[\left(1-f_{s}\right)+\left(1-f_{d}\right)\right] \sigma_{a}^{2}$, where $f_{s}$ and $f_{d}$ are inbreeding coefficients of the sire and dam). The phenotypic value was generated in the same way as the individuals in the base population, assuming $\sigma_{e}^{2}$ to be constant across generations.

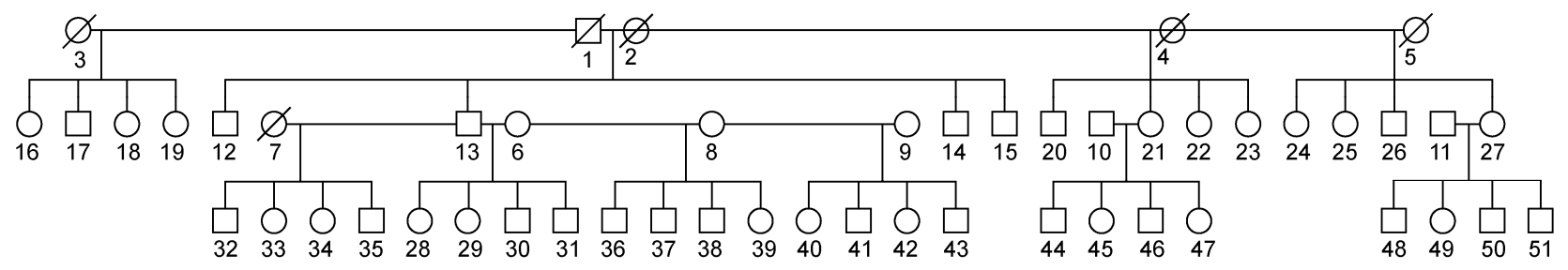

Figure 1 Pedigree structure used in the simulations. 
The QTL effect, expressed as a ratio of QTL variance to the total genetic variance $R_{\mathrm{QTL}}=\sigma_{q}^{2} / \sigma_{G}^{2} \quad\left(\sigma_{G}^{2}=\sigma_{q}^{2}+\sigma_{g}^{2}\right)$, was set at $0.10,0.30$ and 0.50 . The heritability, defined as the ratio of the total genetic variance to the phenotypic variance $h^{2}=\sigma_{G}^{2} / \sigma_{P}^{2} \quad\left(\sigma_{P}^{2}=\sigma_{q}^{2}+\sigma_{g}^{2}+\sigma_{e}^{2}\right)$, was set at 0.1 and 0.3 . The recombination rate $(r)$ between the marker locus and the QTL was set at 0.50 and 0.0001 . Without losing generality, the phenotypic variance was assumed to be 1 .

(iii) Selective genotyping design. Selective genotyping is an efficient experimental design in gene mapping that can decrease the number of genotyped individuals to reduce the genotyping cost and maintain the capability of gene mapping [14]. In selective genotyping, only the individuals in the top two tails are selected; for example, for one pedigree with a selection proportion of 0.2 , the top $10 \%$ of individuals with the highest performance and the bottom $10 \%$ of individuals with the lowest performance were selected and genotyped. In this study, we evaluated the performance of our approach in selective genotyping design by assigning five levels for the selection proportion, namely, 1.0, 0.8, 0.6, 0.4 and 0.2 .

\subsection{Pedigree transmission disequilibrium test for quantitative traits (QPTDT)}

(i) Conversion of quantitative traits to dichotomous traits. For a quantitative trait controlled by a QTL with alleles Q and $\mathrm{q}$, it was assumed that the individuals with high performance carry allele $\mathrm{Q}$, and those with low performance carry allele q. Therefore, the individuals in a pedigree can be classified into two groups: status 1 , individuals that inherit $\mathrm{Q}$ from heterozygous parent and have high phenotypic value; and status 2, individuals that inherit $\mathrm{q}$ from heterozygous parent and have low phenotypic value. Lange and Laird [15] demonstrated that the average of the phenotypic value could be used to classify individuals with higher and lower phenotypic values into status 1 and 2, respectively. In the pedigree structure commonly found in farm animals, full-sib families and mixed families (full-sib and half-sib families) are very prevalent within one pedigree. Therefore we used the averages of full-sib and mixed families as the selection criterion for classifying the quantitative phenotypes of individual animals within a pedigree. For a full-sib family, the animals with a performance higher than the average for this family were classified as status 1 , and those with a performance lower than the average were status 2 . Likewise, the animals in a mixed family were classified into status 1 and 2 according to whether their performance was higher or lower than the average for that family. These two classification criteria were termed full-sib family selection and mixed family selection respectively.

(ii) Pedigree transmission disequilibrium test. The dichotomous status 1 and 2 trait was treated as a disease trait with affected (status 1) and unaffected (status 2) as de- scribed in the original transmission disequilibrium test [2]. According to previous studies, two types of nuclear families can provide linkage disequilibrium information: (1) a family with at least one status 1 offspring, both parents available, and at least one parent heterozygous for the marker being studied; and (2) a family with at least one discordant sibship, and with or without the parental information [5,6,11]. A discordant sibship is defined as a pair of siblings who are different in both phenotypic status and marker genotypes [5]. A pedigree was considered informative if it contained at least one nuclear family of either type. For any one pedigree, let $n_{\mathrm{T}}$ and $n_{\mathrm{S}}$ denote the number of the first and second type nuclear families, respectively. Considering a marker locus with two alleles $M_{1}$ and $M_{2}$, for the first type nuclear families, we defined a variable $X_{T}=(1-u)\left(N_{t a}-N_{n a}\right)-u\left(N_{t u}\right.$ $-N_{n u}$ ), where $u$ is the proportion of status 1 offspring in the population, $N_{t a}$ and $N_{t u}$ are the number of times the heterozygous parents transmit $M_{1}$ to their status 1 and status 2 offspring, respectively, and $N_{n a}$ and $N_{n u}$ are the number of times the heterozygous parents do not transmit $M_{1}$.

Similarly, a variable $X_{S}=N_{s a}-N_{s u}$ was defined for the second type of nuclear families, where $N_{s a}$ represents the number of allele $M_{1}$ in sibs with status 1 , and $N_{s u}$ represents the number of $M_{1}$ in sibs with status 2 .

For a pedigree with $n_{\mathrm{T}}$ first type nuclear families and $n_{\mathrm{S}}$ second type nuclear families, a combined variable was defined as

$$
D=\frac{1}{n_{\mathrm{T}}+n_{S}}\left(\sum_{j=1}^{n_{\mathrm{T}}} X_{T j}+\sum_{j=1}^{n_{S}} X_{S j}\right) .
$$

Under the null hypothesis of no association, $E\left(X_{T}\right)=0$ for all first type of nuclear families and $E\left(X_{S}\right)=0$ for all second type of nuclear families, and consequently, $E(D)=0$. Therefore, for a sample with $N$ independent pedigrees, we get

$$
E\left(\sum_{i=1}^{N} D_{i}\right)=0, \operatorname{Var}\left(\sum_{i=1}^{N} D_{i}\right)=\sum_{i=1}^{N} \operatorname{Var}\left(D_{i}\right)=E\left(\sum_{i=1}^{N} D_{i}^{2}\right),
$$

where $D_{i}$ represents the combined variable for pedigree $i$.

The test statistic for testing the pedigree transmission disequilibrium can be constructed as

$$
T=\sum_{i=1}^{N} D_{i} / \sqrt{\sum_{i=1}^{N} D_{i}^{2}},
$$

which follows asymptotically normal distribution under the null hypothesis of no association.

\subsection{Permutation test}

We used a bi-allelic marker without linkage with the QTL (recombination rate equal to 0.5 ) to evaluate the capacity of our approach in controlling type I error, and another marker with complete linkage with the QTL (recombination rate 
equal to 0.0001 to meet the requirement of QTDT) to evaluate the power of our approach. Strictly speaking, the test statistic that we proposed does not follow normal distribution, therefore, we implemented a permutation test, in which the phenotype value for each individual was permuted while the genotype remained as the original one, to construct the empirical distribution of QPTDT. In this study, 10000 permutations were carried out.

The computing time of the algorithms was measured in minutes on an IBM server (SUSE Linux 9.2 and $3 \mathrm{GHz}$ Intel Xeon processor).

\section{Results}

We carried out a series of simulation studies to evaluate the performance of the proposed QPTDT approach, 100 replicates were simulated in each simulation scenario.

\subsection{Comparisons of conversion methods of quantitative traits}

In the first step quantitative traits were converted to dichotomous traits with status 1 and status 2 , and the two conversion methods, full-sib family selection and mixed family selection were implemented. The efficiency of QPTDT under the two conversion methods is shown in Table 1 . In most of the scenarios tested, mixed family selection performed better than full-sib family selection in terms of power and type I error. When the QTL effect was small, the power of QPTDT with mixed family selection was over $10 \%$ higher than with full-sib family selection. Moreover, mixed family selection controlled the type I error below the pre-assigned significance levels of 0.05 and 0.01 , while the type I error was slightly inflated when the full-sib family selection was used.

\subsection{Efficiency of family size and number of pedigree}

As expected, the efficiency of our approach was improved by increasing the number of offspring per dam or by using as independent extended pedigree. As illustrated in Table 1, when the QTL effect level was 0.1, the power of QPTDT was only 0.57 when ten extended pedigrees and four offspring per dam were used; its power dramatically increased to 0.92 or 0.98 when the extended pedigrees were doubled or when the offspring per dam were increased. This result also indicated that increasing the number of offspring per dam was more effective, probably because more informative nuclear family trios and discordant sibships were produced. This finding is similar to the report by Martin et al. [16] that large pedigree could provide more information to improve the efficiency of the pedigree disequilibrium test.

\subsection{Comparisons of QPTDT with PDT and QTDT}

Several popular programs to deal with pedigree data are available. In our simulation study, we compared the QPTDT approach with two other popular methods QTDT [10] and PDT [6]. QTDT uses a regression model to test associations in quantitative trait analysis [10]. The main difference between PDT and our approach is that the information from both affected and unaffected offspring can be used in our approach while only affected offspring are considered by PDT. Although PDT can deal only with discrete traits, it can be implemented to deal with quantitative traits after converting quantitative phenotypes using mixed family selection, as we have done in QPTDT. Table 2 shows the performance of QTDT, PDT and QPTDT when three different combinations of MAF $q$ (minor allele frequency) and $h^{2}$ were simulated. The results indicated that QPTDT was more robust and powerful than QTDT and PDT in all the tested scenarios; QTDT had the lowest power in detection of the QTL, especially in scenarios with small QTL effects. The power of PDT was close to the power of QPTDT; however, the type I error of PDT was relatively high, resulting in more false positive detections. For instance, the type I error of PDT was 0.18 and 0.15 when the QTL effect was 0.1 , the number of offspring per dam was four, MAF was 0.1 and $h^{2}$ was 0.3 . Generally, the QPTDT approach controlled the type I error below or very close to the significance level in all scenarios and, compared with PDT and QTDT, QPTDT had the highest power in the same scenario.

Table 1 Performance of QPTDT under the conversion methods of full-sib family selection and mixed-family selection with MAF 0.1 and $h^{2} 0.3$ (based on simulations of 100 replicates)

\begin{tabular}{|c|c|c|c|c|c|c|c|c|c|c|}
\hline \multirow{3}{*}{$\begin{array}{c}\text { No. } \\
\text { pedigree }\end{array}$} & \multirow{3}{*}{$\begin{array}{c}\text { No. } \\
\text { offspring/dam }\end{array}$} & \multirow{3}{*}{$\begin{array}{l}\text { QTL } \\
\text { effect }\end{array}$} & \multicolumn{4}{|c|}{$\alpha=0.05$} & \multicolumn{4}{|c|}{$\alpha=0.01$} \\
\hline & & & \multicolumn{2}{|c|}{ Power } & \multicolumn{2}{|c|}{ Type I error } & \multicolumn{2}{|c|}{ Power } & \multicolumn{2}{|c|}{ Type I error } \\
\hline & & & Full-sib & Mixed & Full-sib & Mixed & Full-sib & Mixed & Full-sib & Mixed \\
\hline \multirow{4}{*}{10} & \multirow{3}{*}{4} & 0.1 & 0.44 & 0.59 & 0.08 & 0.01 & 0.20 & 0.30 & 0.02 & 0 \\
\hline & & 0.3 & 0.86 & 0.96 & 0.04 & 0.04 & 0.56 & 0.77 & 0 & 0 \\
\hline & & 0.5 & 0.99 & 1.00 & 0.07 & 0.04 & 0.85 & 0.93 & 0.02 & 0.01 \\
\hline & 8 & 0.1 & 0.98 & 0.99 & 0.05 & 0.03 & 0.82 & 0.85 & 0.01 & 0.01 \\
\hline \multirow{2}{*}{20} & \multirow{2}{*}{4} & 0.1 & 0.80 & 0.92 & 0.05 & 0.05 & 0.56 & 0.71 & 0.03 & 0.01 \\
\hline & & 0.3 & 1.00 & 1.00 & 0.07 & 0.06 & 0.99 & 0.99 & 0.02 & 0.01 \\
\hline
\end{tabular}


Table 2 Comparisons of the performances of QTDT, PDT and QPTDT in three different scenarios (based on simulations of 100 replicates)

\begin{tabular}{|c|c|c|c|c|c|c|c|c|c|c|c|c|c|c|}
\hline \multirow{3}{*}{$\begin{array}{c}\text { No. } \\
\text { pedigree }\end{array}$} & \multirow{3}{*}{$\begin{array}{c}\text { No. } \\
\text { offspring/dam }\end{array}$} & \multirow{3}{*}{$\begin{array}{l}\text { QTL } \\
\text { effect }\end{array}$} & \multicolumn{6}{|c|}{$\alpha=0.05$} & \multicolumn{6}{|c|}{$\alpha=0.01$} \\
\hline & & & \multicolumn{3}{|c|}{ Power } & \multicolumn{3}{|c|}{ Type I error } & \multicolumn{3}{|c|}{ Power } & \multicolumn{3}{|c|}{ Type I error } \\
\hline & & & QTDT & PDT & QPTDT & QTDT & PDT & QPTDT & QTDT & PDT & QPTDT & QTDT & PDT & QPTDT \\
\hline \multicolumn{15}{|c|}{ MAF: $0.5, h^{2}: 0.3$} \\
\hline \multirow{3}{*}{10} & \multirow{2}{*}{4} & 0.1 & 0.50 & 0.85 & 0.96 & 0.12 & 0.14 & 0.04 & 0.26 & 0.50 & 0.67 & 0.02 & 0.04 & 0.01 \\
\hline & & 0.3 & 0.95 & 1.00 & 1.00 & 0.27 & 0.14 & 0.05 & 0.91 & 0.91 & 0.97 & 0.15 & 0.04 & 0.00 \\
\hline & 8 & 0.1 & 0.98 & 1.00 & 1.00 & 0.32 & 0.04 & 0.03 & 0.87 & 0.99 & 1.00 & 0.14 & 0.00 & 0.00 \\
\hline 20 & 4 & 0.1 & 0.86 & 1.00 & 1.00 & 0.21 & 0.09 & 0.05 & 0.64 & 0.99 & 1.00 & 0.06 & 0.02 & 0.01 \\
\hline \multicolumn{15}{|c|}{ MAF: $0.1, h^{2}: 0.3$} \\
\hline \multirow{3}{*}{10} & \multirow{2}{*}{4} & 0.1 & 0.18 & 0.46 & 0.59 & 0.04 & 0.18 & 0.01 & 0.08 & 0.19 & 0.30 & 0 & 0.01 & 0 \\
\hline & & 0.3 & 0.54 & 0.94 & 0.96 & 0.12 & 0.04 & 0.04 & 0.33 & 0.75 & 0.77 & 0.03 & 0 & 0.01 \\
\hline & 8 & 0.1 & 0.53 & 0.94 & 0.99 & 0.15 & 0.06 & 0.03 & 0.36 & 0.78 & 0.85 & 0.02 & 0.01 & 0.01 \\
\hline 20 & 4 & 0.1 & 0.41 & 0.85 & 0.92 & 0.08 & 0.15 & 0.05 & 0.18 & 0.54 & 0.71 & 0.01 & 0.03 & 0.01 \\
\hline \multicolumn{15}{|c|}{ MAF: $0.1, h^{2}: 0.1$} \\
\hline \multirow[t]{2}{*}{20} & 4 & 0.05 & 0.16 & 0.45 & 0.55 & 0.03 & 0.16 & 0.06 & 0.09 & 0.21 & 0.29 & 0.01 & 0.02 & 0.00 \\
\hline & & 0.1 & 0.36 & 0.76 & 0.82 & 0.09 & 0.04 & 0.05 & 0.20 & 0.53 & 0.62 & 0.01 & 0.02 & 0.00 \\
\hline
\end{tabular}

Our simulation study showed that minor allele frequency at the QTL influenced the performance of all three approaches, especially when the QTL effect was smaller. The power of QTDT, PDT and QPTDT decreased as the MAF decreased from 0.5 to 0.1 . This decrease in power was particularly severe for QTDT; its power was decreased by nearly $50 \%$ in most scenarios. The heritability similarly affected the performance of QTDT, PDT and QPTDT as the power of the three approaches decreased when $h^{2}$ was decreased from 0.3 to 0.1 . Further, the three tests were not good at detecting small effect genes; for example, when the QTL effect was 0.05 and $h^{2}$ was 0.1 , the QTL variance was as low as $0.5 \%$ (Table 2), indicating that the power of QTDT, PDT and QPTDT was very low.

The average computing times of QTDT, PDT and QPTDT for one replicate are shown in Table 3 . The results show that QTDT was the slowest mostly because a maximum likelihood method is implemented in QTDT. Particularly, QTDT took nearly $1.5 \mathrm{~h}$ to simulate the scenario of eight offspring per dam; PDT and QPTDT took only several seconds to simulate the same scenario.
Table 3 Average computing time (min) of QTDT, PDT and QPTDT for one replicate (MAF 0.1 and $h^{2} 0.3$ )

\begin{tabular}{cccccc}
\hline $\begin{array}{c}\text { No. } \\
\text { pedigree }\end{array}$ & $\begin{array}{c}\text { No. } \\
\text { offspring/dam }\end{array}$ & $\begin{array}{c}\text { QTL } \\
\text { effect }\end{array}$ & QTDT & PDT & QPTDT \\
\hline \multirow{2}{*}{10} & 4 & 0.1 & 31.78 & 0.02 & 0.06 \\
& 8 & 0.3 & 16.88 & 0.02 & 0.04 \\
& & 0.1 & 99.85 & 0.01 & 0.03 \\
\hline 20 & 4 & 0.1 & 47.45 & 0.06 & 0.15 \\
\hline
\end{tabular}

\subsection{Performance of QPTDT in selective genotyping design}

We simulated a series of scenarios to evaluate our QPTDT approach in selective genotyping design. The selection proportions were assigned as 1.0, 0.8, 0.6, 0.4 and 0.2. As shown in Table 4, in general, selective genotyping improved the efficiency of QPTDT compared with full genotyping (selection proportion equal to 1.0). Compared with the QTDT and PDT methods, our approach had equal or higher power of detecting QTL with a type I error that was below the significance level in most situations. For large pedigrees,

Table 4 Performance of QPTDT in selective genotyping design with MAF 0.5 and $h^{2} 0.3$ and a QTL effect of 0.1 (based on simulations of 100 replicates)

\begin{tabular}{|c|c|c|c|c|c|c|}
\hline \multirow{2}{*}{ No. pedigree } & \multirow{2}{*}{ No. offspring/dam } & \multirow{2}{*}{ Selection proportion } & \multicolumn{2}{|c|}{ Power } & \multicolumn{2}{|c|}{ Type I error } \\
\hline & & & $\alpha=0.05$ & $\alpha=0.01$ & $\alpha=0.05$ & $\alpha=0.01$ \\
\hline \multirow{5}{*}{10} & \multirow{5}{*}{8} & 1.0 & 0.99 & 0.85 & 0.03 & 0.01 \\
\hline & & 0.8 & 0.99 & 0.84 & 0.05 & 0.02 \\
\hline & & 0.6 & 1.00 & 0.91 & 0.02 & 0 \\
\hline & & 0.4 & 0.98 & 0.85 & 0.06 & 0.01 \\
\hline & & 0.2 & 0.91 & 0.69 & 0.06 & 0 \\
\hline \multirow{5}{*}{20} & \multirow{5}{*}{4} & 1.0 & 0.92 & 0.71 & 0.05 & 0.01 \\
\hline & & 0.8 & 0.92 & 0.75 & 0.04 & 0.01 \\
\hline & & 0.6 & 0.90 & 0.65 & 0 & 0 \\
\hline & & 0.4 & 0.87 & 0.59 & 0.05 & 0 \\
\hline & & 0.2 & 0.54 & 0.22 & 0.01 & 0.01 \\
\hline
\end{tabular}


for example, those with eight offspring per dam, the power of QPTDT at a selection proportion of 0.6 was 1.00 and 0.91 at significance levels of 0.05 and 0.01 , respectively. This is higher than the power obtained with full genotyping. When the selection proportion was 0.8 or 0.4 , the selective genotyping performance of QPTDT was similar to its performance with full genotyping. For small pedigrees, for example, those with four offspring per dam, the power of QPTDT at a selection proportion of 0.80 was close to that of full genotyping. However, its power decreased when less than $60 \%$ individuals were selected for genotyping. This finding indicated that large pedigrees were required for selective genotyping design because they provided enough informative nuclear families or discordant sibships even when only some proportion of individuals were genotyped. For small pedigree, when perhaps there were no genotyped individuals in some informative nuclear families or no discordant sibships, the power of QPTDT was significantly reduced.

\section{Discussion}

Ewens et al. [17] summarized the similarities and differences of the various TDTs in a literature review. The original TDT and most of its extensions were focused mainly on discrete traits, while many genetic association studies were concerned with quantitative traits, meaning that pedigree information is becoming more and more important. In an extension of TDT to handle pedigree information, Chen et al. [18] proposed a generalized disequilibrium test (GDT) to generalize TDT-like family-based association methods. This method assesses the genotype difference of all discordant relative pairs in a family and makes use of information beyond first-degree relative pairs. While it still aims to deal with dichotomous traits, its performance in dealing with quantitative traits is unknown. In this paper, we proposed a new approach, QPTDT, to handle quantitative traits. In addition to the conversion of quantitative traits to discrete ones, the kernel of our approach is a modification of the pedigree disequilibrium test (PDT) proposed by Martin et al. [6]. PDT only makes use of information from affected offspring in nuclear families because, like the original TDT, it has mainly been applied to investigate rare diseases. However, for common disorders, unaffected offspring can also contribute information [12]. We have used the information from both affected and unaffected offspring in the QPTDT approach. The results of our simulation studies show that the power of the test can be improved and false positive detections can be controlled by taking both affected and unaffected statuses into account. In most of the simulated situations, our approach outperformed PDT.

One possible novelty of our approach is the conversion of quantitative traits to binary traits. This concept arose mainly from the knowledge that TDT is more powerful in dealing with dichotomous traits [2]; however, splitting continuous data into two discrete groups risks the loss of information which could result in the substantial loss of power. To assess this possibility, we compared our approach with the general linear model-based method QTDT [10] in which a regression model is used to test association in quantitative trait analysis. Compared with QTDT, our approach performed better with higher power and lower type I error, especially in the scenarios of small QTL effects and low MAF. Lange and Laird [15] treated sibs in the top 10\% tail as affected and those in the lower $10 \%$ tail as unaffected in their simulation study of quantitative trait, and found that program FBAT and PDT approach performed better than QTDT. Moreover, QTDT requires a lot more computer time than the other methods, which makes its use prohibitive especially when dealing with a large number of makers. Instead of using the method of Lange and Laird [15] to assign status, we used the average of the phenotypic value to group all individuals into either status 1 or status 2; some individuals with phenotypic values close to the average might easily be assigned the wrong status. To overcome this possibility, we implemented a selective genotyping design in QPTDT and only individuals in the top and lower tails were assigned statuses. Our results showed that selective genotyping improved the performance of our approach.

Laird and Lange [1] reported that the allele frequency of a causative gene affected the power of the case-control and trios designs; in general, low power occurred when there was low MAF in the causative gene. Our study confirmed this point. The QTL effect is another important factor that influences the performance of QTDT, PDT and QPTDT. Our study demonstrated that the three tests were not so powerful in detecting small gene effects. In future studies, it will be challenging to modify these methods to study quantitative traits underlying small genetic effects.

We evaluated the performance of our approach in selective genotyping design. For large pedigrees, selective genotyping design performed as well as or better than full genotyping design when more than $40 \%$ of the individuals in the two tails were genotyped. Therefore, it is reasonable to apply a selective genotyping strategy in the QPTDT approach that we have proposed. However, for small pedigrees, the selective genotyping designs only performed as well as or better than the full genotyping design when more than $80 \%$ of the individuals in the two tails were genotyped. Selective genotyping design assumes that individuals with the higher performance carry allele Q, while those with the lower performance carry allele q. This assumption is in accordance with the classification of the quantitative phenotypes into two categories. The accuracy of the classification can be improved when only individuals in the higher and lower tails are selected to be genotyped, because, when all the individuals are used, those with phenotypes close to the average may carry either allele Q or q, leading to their possible misclassification. The transmission disequilibrium test 
is seriously affected by misclassification [1]. A similar study was conducted by Lange and Laird [15] who compared the performance of program FBAT when different sample proportions were chosen. They found that when the scenario of top $10 \%$ tail and lower $10 \%$ tail was used, FBAT had the highest power; the power reduced with the top $10 \%$ tail and lower $30 \%$ tail, and with the full genotyping sample.

Our simulation study also implied that selective genotyping was more effective for large pedigrees. For small pedigrees, although selective genotyping improved the accuracy of the classification, it probably reduced the number of informative nuclear families and discordant sibships. It is difficult to select the best balance point for implementation of selective genotyping. According to our study, it is not recommended to apply selective genotyping to pedigrees with an average of less than four offspring per dam, because the number of available informative families may not be enough to produce accurate results.

In this study, single marker analysis was carried out. For multiple tightly-linked markers, two options are currently available: the haplotype-based test and the multiple-single marker test. Tightly-linked markers could be regarded as a single marker because only a few recombination events occur among them and the haplotypes could be handled as alleles. In haplotype-based tests multiple markers are analyzed simultaneously which provides more genetic information; therefore, this test should be more powerful than single-point analysis [19]. However, some researchers have argued that single marker analysis is comparable to multiple marker analysis [20]. QPTDT can be extended for haplotype-based analysis. In this study, we have developed a test statistic for markers (e.g. SNPs) with only two alleles. For haplotype-based analysis, the Zmax approach [5] can be applied in QPTDT. Suppose that there are $k$ haplotypes (H1, $\mathrm{H} 2, \ldots, \mathrm{H} k$ ) for multiple linked markers. If the transmission of haplotype $\mathrm{H} 1$ is considered, then $\mathrm{H} 1$ is assumed to be one allele and all other haplotypes are grouped as 'non-H1'. QPTDT can now be performed in the same way as for a single SNP. This procedure should be repeated for each of the $k-1$ haplotypes, and then the test statistic with the largest absolute value (Zmax) among the $k$ values is chosen as the final test statistic. Alternatively, a multiple singlemarker test could be used for multiple marker analysis; however, a series of demanding issues such as numerous hypothesis-testing would need to be explored.

In farm animals, large half-sib families (e.g. in cattle) or full-sib families (e.g. in pigs) and overlapping generations are common. Therefore, extended pedigrees can be easily obtained. An attractive advantage of our QPTDT approach is its ability to handle population admixture and population stratification which are usually important sources of false positive associations. The QPTDT approach can be improved when data from different sources, for example, different breeds or different lines in farm animals are used.

This work was supported by the Scientific Research Foundation for Returned Scholars, Ministry of Education of China, National Natural Science Foundation of China (30800776), the Program for Changjiang Scholar and Innovation Research Team in University (IRT1191), the Beijing Science and Technology Ministry Project (Z101105000810001) and the "948" of Ministry of Agriculture Project, China (2011-G2A).

1 Laird N M, Lange C. Family-based designs in the age of large-scale gene-association studies. Nat Rev Genet, 2006, 7: 385-394

2 Spielman R S, Mcginnis R E, Ewens W J. Transmission test for linkage disequilibrium: The insulin gene region and insulin-dependent diabetes mellitus (IDDM). Am J Hum Genet, 1993, 52: 506-516

3 Martin E R, Kaplan N L, Weir B S. Test for linkage and association in nuclear families. Am J Hum Genet, 1997, 61: 439-448

4 Horvath S, Laird N M. A discordant-sibship test for disequilibrium and linkage: No need for parental data. Am J Hum Genet, 1998, 63: 1886-1897

5 Spielman R S, Ewens W J. A sibship test for linkage in the presence of association: The sib transmission/disequilibrium test. Am J Hum Genet, 1998, 62: 450-458

6 Martin E R, Monks S A, Warren L L, et al. A test for linkage and association in general pedigrees: The pedigree disequilibrium test. Am J Hum Genet, 2000, 67: 146-154

7 Allison D B. Transmission-disequilibrium tests for quantitative traits. Am J Hum Genet, 1997, 60: 676-690

8 Rabinowitz D. A transmission disequilibrium test for quantitative trait loci. Hum Hered, 1997, 47: 342-350

9 Sun F, Flanders W D, Yang Q, et al. Transmission/disequilibrium tests for quantitative traits. Ann Hum Genetics, 2000, 64: 555-565

10 Abecasis G R, William O C, Cardon L R. Pedigree tests of transmission disequlibrium. Eur J Hum Genet, 2000, 8: 545-551

11 Lunetta K L, Faraone S V, Biederman J, et al. Family-based tests of association and linkage that use unaffected sibs, covariates, and interactions. Am J Hum Genet, 2000, 66: 605-614

12 Silverman E K, Shapiro S D, Lomas D A, et al. Respiratory Genetics. Boston: Hodder Arnold, 2005. 27-46

13 Falconer D S, Mackay T F C. Introduction to Quantitative Genetics. 4th ed. London: Longman, 1996

14 Darvasi A, Soller M. Selective genotyping for determination of linkage between a marker locus and a quantitative trait locus. Theor Appl Genet, 1992, 85: 353-335

15 Lange C, Laird N M. On a general class of conditional tests for family-based association studies in genetics: The asymptotic distribution, the conditional power and optimality considerations. Genetic Epidemiol, 2002, 23: 165-180

16 Martin E R, Bass M P, Kaplan N L. Correcting for a potential bias in the pedigree disequilibrium test. Am J Hum Genet, 2001, 68: 10651067

17 Ewens W J, Li M, Spielman R S. A review of family-based tests for linkage disequilibrium between a quantitative trait and a genetic marker. PLoS Genetics, 2008, 4: e1000180

18 Chen W M, Manichaikul A, Stephen S R. A generalized family-based association test for dichotomous traits. Am J Hum Genetics, 2009, 85: 364-376

19 Zhao H, Zhang S, Merikangas $\mathrm{K}$ R, et al. Transmission/ disequilibrium tests for multiple tightly linked markers. Am J Hum Genet, 2000, 67: 936-946

20 Grapes L, Dekkers J C M, Rothschild M F, et al. Comparing linkage disequilibrium-based methods for fine mapping quantitative trait loci. Genetics, 2004, 166: 1561-1570

Open Access This article is distributed under the terms of the Creative Commons Attribution License which permits any use, distribution, and reproduction in any medium, provided the original author(s) and source are credited. 